\title{
Flow-Injection Electrogenerated Chemiluminescence Determination of Isoniazid Using Luminol
}

\author{
Xingwang Zheng, Zhihui Guo, and Zhujun ZHANG ${ }^{\dagger}$ \\ Department of Chemistry, Shaanxi Normal University, Xi'an 710062, P. R. China
}

\begin{abstract}
Based on a new electrogenerated chemiluminescence (ECL) analytical idea, this paper explains a sensitive and selective flow-injection ECL method using luminol for the determination of isoniazid, based on the sensitizing effect of isoniazid for the weak ECL emission of electrochemically oxidized luminol. Under the optimum experimental conditions, the relative ECL intensity was linear with isoniazid concentration in the range of $4.0 \times 10^{-8} \mathrm{~mol} / \mathrm{L}$ to $8.0 \times 10^{-6} \mathrm{~mol} / \mathrm{L}$ and with a detecting limit of $2.8 \times 10^{-8} \mathrm{~mol} / \mathrm{L}$.
\end{abstract}

(Received March 15, 2001; Accepted June 29, 2001)

\section{Introduction}

Isoniazid (pyridine-4-carboxylic acid hydrazide) is an important drug compound for the chemotherapy of tuberculosis. Many analytical techniques, ${ }^{1-5}$ including spectrometric, ${ }^{1,2}$ fluorescence, ${ }^{3,4}$ electroanalytical ${ }^{5}$ and flow-injection chemiluminescence methods, ${ }^{6,7}$ have been developed for its determination. For example, based on the sensitizing effect of isoniazid for the $\mathrm{CL}$ reaction of $\mathrm{ClO}^{-}$with luminol, a more sensitive CL method for isoniazid has been developed by Huang et al. and a $4.6 \times 10^{-8} \mathrm{~mol} / \mathrm{L}$ isoniazid can be detected. However, due to the strong background CL emission arising from the chemical reaction of $\mathrm{ClO}^{-}$with luminol, the higher sensitivity for isoniazid is hampered. On the other hand, due to the significant pharmaceutic functions of isoniazid, there is still a need for rapid, inexpensive, but more sensitive and selective method for the determination of isoniazid, especially in the in vivo blood samples, to assess its pharmacokinetic performance.

More recently, as a useful sampling technique, microdialysis (MD) in vivo sampling technique has been extensively used in analytical science. In generally, microdialysis provides the clean samples that do not require any clean-up procedure prior to analysis, and the microdialysis probes can also be implanted in many tissues ${ }^{9-12}$ (such as in the brain ${ }^{9,10}$ and in the blood ${ }^{11,12}$ ) with minimal discomfort to the experimental animal. So this sampling technique may be a better candidate to sample isoniazid in the in vivo blood sample directly. But up to now, no report treats the on-line combination of isoniazid detecting method reported with $\mathrm{MD}$ in vivo sampling technique for analytical purposes.

As a powerful analytical tool, electrogenerated chemiluminescence (ECL) analysis has promising advantages over conventional CL. ${ }^{13-17}$ First, the ECL reaction is easily regulated and manipulated by the electrode potential. Secondly, the luminescence is localized on the electrode surface, which allows the design of an efficient measurement for ECL signal. Thirdly, luminescence is electrochemically generated in situ

$\dagger$ To whom correspondence should be addressed.

E-mail: zzj18@hotmail.com without any contamination due to the addition of analytical reagents. Up to now, many useful ECL systems have been developed and have been widely used in analytical sciences. ${ }^{15-17}$ However, in the reported ECL systems, ${ }^{13,15}$ it is clear that the most promising and rapidly developing area of ECL analysis concerns the luminol ECL system. ${ }^{18-22}$ This is because this ECL system presents the high ECL emission quantum yields, the lower oxidizing potential and the inexpensive reagent consumption. In addition, the luminol-based ECL reaction can occur in aqueous buffered solutions in the presence of oxygen and other impurities. In the luminol-based ECL analysis, many analytes, such as hydrogen peroxide,${ }^{23}$ glucose and choline, ${ }^{24,25}$ metal ions $\left(\mathrm{Co}^{2+}, \mathrm{Cu}^{2+}, \mathrm{Ni}^{2+} \text { etc. }\right)^{26-28}$ and some luminol-labeled compounds, ${ }^{29-31}$ have been sensitively detected. But no paper concerns any directly enhanced effect of organic compound on the weak ECL emission of luminol for analytical purposes.

In this research, we found that, at $0.80 \mathrm{~V}(v s . \mathrm{Ag} / \mathrm{AgCl}$ reference electrode) electrolytic potential, the weak ECL signal which was generated from electrochemical oxidizing luminol on the surface of platinum electrode in the borax buffer medium was greatly enhanced by isoniazid. The ECL emission increment was related to the amount of isoniazid. Based on this observation, a new sensitive and selective flow-injection ECL method for the determination of isoniazid was firstly described. In addition, because the ratio of the enhancing ECL signal of isoniazid to the background ECL signal of electrochemical oxidizing luminol can be adjusted and further optimized by selecting the suitable electrolytic potential for use in the proposed ECL method, the sensitivity of the proposed ECL method for the analysis of isoniazid, was superior to with those in other methods for isoniazid. ${ }^{6-8}$ At the same time, according to the possible ECL reaction mechanism proposed, a new idea, based on coupling in situ electrochemical oxidizing reaction of analyte with the reducing reaction of the dissolving oxygen to generate active $\mathrm{CL}$ reaction species to achieve analysis of analyte, was also developed. At last, it was further found that this ECL method can be coupled effectively with MD in vivo sampling technique to in vivo detect the change of the isoniazid concentration in the blood of the rabbit to assess its pharmacokinetic performance. 


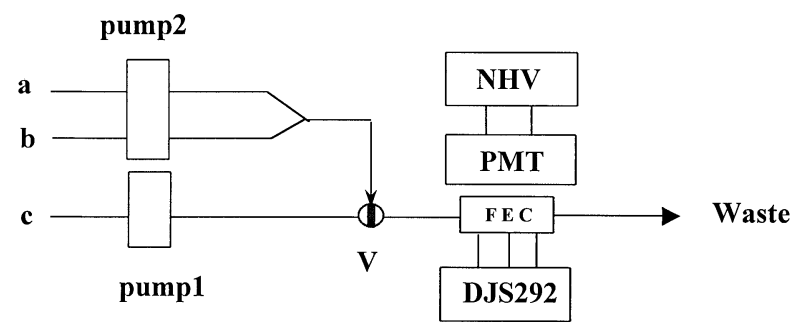

Fig. 1 The schematic diagram of the flow-injection ECL system. a, sample stream; b, luminol reagent stream; c, borax buffer solution carrier stream; FEC, flow-through electrolytic cell; DJS292, potentiostat; V, six-way injection valve; pump 1 and pump 2, peristaltic pump; PMT, R456 photomultiamplier tube; NHV, negative high voltage.

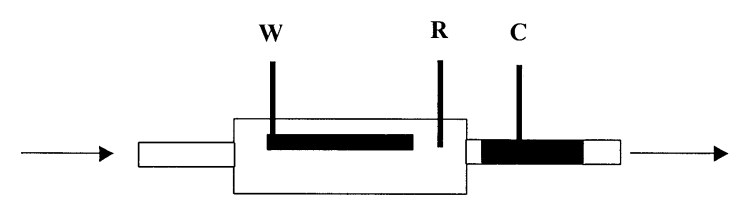

Fig. 2 The structure of the flow-through electrolytic cell. W, working electrode; R, reference electrode; C, counter electrode.

\section{Experimental}

\section{Reagents}

All solutions were prepared from analytical-reagent grade materials with distilled, deionized water. Stock isoniazid solution $(100.0 \mu \mathrm{mol} / \mathrm{L})$ was obtained by dissolving suitable weight of isoniazid (Shanghai Chemical reagent plant) in water, and then transferring this solution into a calibrated flask and diluting to $1 \mathrm{~L}$ with water. The $0.05 \mathrm{~mol} / \mathrm{L}$ borax buffer stock solution ( $\mathrm{pH}=9.26$ ) was made by dissolving $9.7 \mathrm{~g}$ of $\mathrm{Na}_{2} \mathrm{~B}_{4} \mathrm{O}_{7}$ in $0.5 \mathrm{~L}$ water.

\section{Apparatus}

The manifold scheme of the ECL flow system used in this work is shown in Fig. 1. Two peristaltic pumps were used to deliver the carrier stream (borax buffer solution), sample solution and luminol solution. The injections of both the mixing solution of sample with luminol and the luminol solution was made using a six-port valve equipped with a $30 \mu \mathrm{L}$ sample loop. The flow-through electrolytic cell (FEC) utilizes a conventional three-electrode setup and was arranged as shown in Fig. 2. The flow-cell was made of glass tubing (length: 3.0 $\mathrm{cm}$; i.d.: $3 \mathrm{~mm}$ ). The working electrode was a platinum flake (length: $2.0 \mathrm{~cm}$; width: $3 \mathrm{~mm}$ ). The stainless-steel tube and $\mathrm{Ag} / \mathrm{AgCl}$ wire were used as the counter electrode and reference electrode, respectively. The distance between the six-port valve and the FEC was $10 \mathrm{~cm}$ as in Fig. 1. The applied potential for constant potential electrolysis was achieved by a DJS292 potentiostat/galvanostat (Shanghai analytical instrument plant). The ECL intensity was transformed into an electrical signal by an R456 photomultiplier (Hamamatsu) which was operated at $-800 \mathrm{~V}$ and was directly placed in front of the ECL flow-cell. The signal was recorded with a Petinum II computer equipped with an IFFL recording soft (Xi'an Ruike Electronic Company, China).

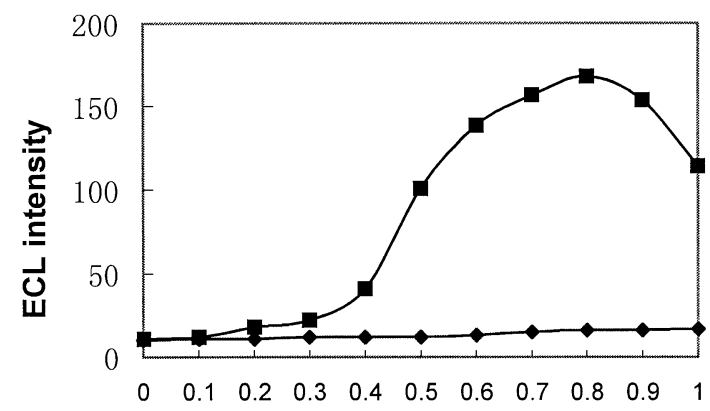

Applied potential (V,vs $\mathrm{Ag} / \mathrm{AgCl}$ reference electrode)

Fig. 3 Effect of the applied potentials on the ECL intensities. Luminol, $4 \times 10^{-6} \mathrm{~mol} / \mathrm{L}$; carrier stream, $0.05 \mathrm{~mol} / \mathrm{L}$ borax buffer solution; isoniazid, $4.0 \times 10^{-7} \mathrm{~mol} / \mathrm{L}$; $\bullet$, the blank ECL intensities; the enhancing ECL intensities.

\section{Procedures}

In order to achieve good mechanical and thermal stability, the instruments were allowed to run for $10 \mathrm{~min}$ before the first measurement was made. The carrier stream and sample stream were each fed at a flow rate of $2.5 \mathrm{ml} / \mathrm{min}$. Electrolytic potential of $0.80 \mathrm{~V}$ was applied to the working electrode with a DJS292 potentiostat/galvanostat.

While the blank solution and the standard isoniazid solutions or the isoniazid sample solution were injected into the carrier stream with a six-port valve, the blank ECL signal or the enhanced ECL signals were recorded. The concentration of isoniazid was quantified by the peak height of the enhancing ECL emission intensity, which is obtained by deducting the blank ECL intensity of luminol solution from that of the sample or standard isoniazid solutions.

\section{Results and Discussion}

\section{ECL reaction mechanism}

For obtaining a clear ECL reaction scheme on this sensitizing kind of luminol-based ECL reaction, the ECL emission spectrum was taken with an Rf-540 fluorescence spectrophotometer. The procedure was as follows: the flowthrough electrolytic cell was firstly placed on the window of the detector of the Rf-540 fluorescence spectrophotometer and the exciting lamp was turned off, then the luminol solutions which contains isoniazid in the borax buffer medium was made to flow continuously through the flow-through electrolytic cell. After these operations, $0.80 \mathrm{~V}$ electrolytic potential was applied to the working electrode and the ECL emission spectrum was recorded. The result showed that the maximum emission wavelength of the ECL emitter was nearly $425 \mathrm{~nm}$. This indicated that the possible ECL emitter was the excited state of 3-aminophthalate. ${ }^{32}$

The effect of the potential used on the enhancing ECL signal (as shown in Fig. 3) showed that: If no suitable potential was applied to the working electrode, no CL signal was observed; while the potential was at $0.30 \mathrm{~V}$ ( $v s . \mathrm{Ag} / \mathrm{AgCl}$ electrode) but not the typical ECL oxidization potential of luminol, the enhancing ECL signal arising from the isoniazid could be easily observed. Up to $0.80 \mathrm{~V}$, the enhancing ECL signal achieved its maximum value. Above $0.80 \mathrm{~V}$, this enhancing function decreased slowly. At the same time, the blank ECL signal arising from the luminol did not change over the used potential 
Table 1 The effect of the medium of the carrier stream on the ECL intensity for $4.0 \times 10^{-7} \mathrm{~mol} / \mathrm{L}$ isoniazid

\begin{tabular}{lccccc}
\hline $\begin{array}{l}\text { Medium of the carrier } \\
\text { stream }\end{array}$ & $\begin{array}{c}0.1 \mathrm{~mol} / \mathrm{L} \\
\mathrm{NaOH}\end{array}$ & $\begin{array}{c}0.05 \mathrm{~mol} / \mathrm{L} \\
\text { Borax }\end{array}$ & $\begin{array}{c}0.1 \mathrm{~mol} / \mathrm{L} \\
\mathrm{NaHCO}_{3}\end{array}$ & $\begin{array}{c}0.1 \mathrm{~mol} / \mathrm{L} \\
\mathrm{Na}_{2} \mathrm{CO}_{3}\end{array}$ & $\begin{array}{c}0.02 \mathrm{~mol} / \mathrm{L} \\
\mathrm{NH}_{3}-\mathrm{NH}_{4}^{+}\end{array}$ \\
\hline Enhancing ECL signal & 108 & 98 & 37 & 47 & 33 \\
Blank ECL signal & 104 & 8 & 2 & 11 & 21 \\
\hline
\end{tabular}

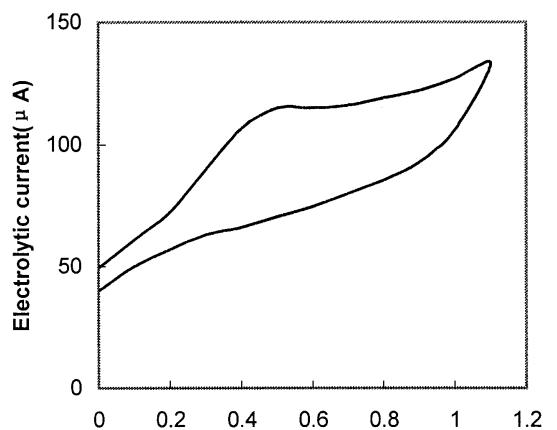

Potential (v, vs $\mathrm{Ag} / \mathrm{AgCl}$ reference electrode)

Fig. 4 The cyclic voltammogram of isoniazid. Isoniazid concentration, $1.0 \times 10^{-3} \mathrm{~mol} / \mathrm{L}$; medium, $0.05 \mathrm{~mol} / \mathrm{L}$ borax buffer solution; scan rate, $50 \mathrm{mV} / \mathrm{S}$.

range. These results suggested that the electrochemically oxidizing product of isoniazid at a suitable oxidizing potential was the key species for the appearance of enhancing ECL signal. Moreover, the cyclic voltammetry experiments of isoniazid (as shown in Fig. 4) in the same experimental conditions as those of its ECL analysis showed that one oxidizing wave related to the electrochemical oxidation of isoniazid can be obviously observed at $0.50 \mathrm{~V}$. This oxidizing potential value was lower than that of the optimum enhancing ECL potential in the flow-injection system. This result further indicated that the key species in the proposed ECL reaction, along with the electrochemically oxidizing products of isoniazid, should include the electrochemically oxidizing products of luminol at $0.80 \mathrm{~V}$.

For presenting the real key electroactive group in the molecule of isoniazid, we further found that the $\mathrm{N}_{2} \mathrm{H}_{4} \cdot \mathrm{H}_{2} \mathrm{SO}_{4}$ presented a similar enhancing ECL function to that of isoniazid for the luminol weak ECL emission. These results indicated that the hydrazine group in the isoniazid was the key enhancing ECLactive function group.

At the same time, we further found that, when the all solutions in this ECL system were deoxidized with pure nitrogen gas, the enhancing function of isoniazid for this weak ECL signal or the weak ECL signals nearly disappeared. This result suggested that the dissolving oxygen in the solutions was another key species in the proposed ECL system.

For exploring the relationship of these two key species (dissolving oxygen and the electrochemically oxidation product of isoniazid at $0.80 \mathrm{~V}$ electrode potential) in the proposed enhancing ECL system, some active oxygen cleaning reagents such as ascorbic acid or benzophenone were added to the solutions of isoniazid or luminol. The experimental results showed that the enhancing ECL signal or ECL signal nearly disappeared when the ascorbic acid was added. These results suggested that the active oxygen, more possible the superoxygen anion radical, may be produced in the proposed ECL reaction.
Based on these investigated results and the chemical property of isoniazid, we presumed that the ECL reaction mechanism of this system was as follows:

At first, both the luminol and isoniazid can be oxidized at 0.80 $\mathrm{V}$ potential on the surface of the platinum flake electrode. The luminol radical and the electrochemically oxidizing products of the isoniazid, an reducing intermediate, were produced, in the diffusion layer of the electrode. This reducing intermediate was most likely the isoniazid radical and thus presented the stronger reducing ability. Then, it can further reduce dissolving oxygen in the solution to generate the superoxygen anion radical. Then, the chemical reaction of the superoxygen anion radical with luminol radical emitted the stronger light.

\section{The design of the flow-injection ECL system}

For obtaining the strongest enhancing ECL signal arising from the isoniazid for this weak ECL signal from the electrochemically oxidizing luminol, many different kinds of designs were suggested for the flow-injection scheme diagrams. The investigated results showed that when the mixing solution of isoniazid with luminol was injected into the flow-through electrolytic cell, the enhancing ECL signal arising from the isoniazid for that of luminol was the strongest. One possible reason may be that the active oxygen was more instable and needed to be in situ provided. Thus, this flow-injection flow system diagram (as shown in Fig. 1) was used in the subsequent experiments.

\section{The selecting of the carrier stream}

The component of the carrier stream, including its $\mathrm{pH}$ and the kinds of the supporting electrolytes, was not only the medium of the ECL reaction of isoniazid, but also the key factor which affected the reproducibility of this ECL analytical method. This was because the carrier stream was also used as the treating solution to treat the working electrode to eliminate the problem of the electrode fouling encountered with constant potential electrolysis methods during the period of sampling times.

Based on this consideration, many different carrier streams such as $\mathrm{NaOH}, \mathrm{Na}_{2} \mathrm{CO}_{3}, \mathrm{NaHCO}_{3}$ and $\mathrm{NH}_{3} \cdot \mathrm{H}_{2} \mathrm{O}$ etc. were used for this purpose in the suitable concentration range; the results investigated are shown in Table 1. As shown in Table 1, although the $0.10 \mathrm{~mol} / \mathrm{L} \mathrm{NaHCO}_{3}$ solution offered the biggest ratio of signal to noise, the reproducibility of the enhancing ECL signal was very poor. At the same time, while $0.05 \mathrm{~mol} / \mathrm{L}$ borax buffer solution was used as the carrier stream, the proposed ECL method presented not only the higher ratio of enhancing ECL signal of isoniazid to blank ECL signal of luminol, but also offered the better reproducibility for monitoring isoniazid. Thus, this buffer solution was selected as the carrier stream for the determination of isoniazid in the proposed ECL system.

\section{Effect of the flow rates}

Based on the principles of flow-injection analysis (FIA) and our preliminary testing, it was found that if the flow rate of the luminol stream was equal to that of the sample (or the standard 
Table 2 The tolerable concentration ratios with respect to isoniazid for some interfering species

\begin{tabular}{lc}
\hline \multicolumn{1}{c}{ Substance } & $\begin{array}{c}\text { Tolerable } \\
\text { concentration ratio }\end{array}$ \\
\hline $\mathrm{K}^{+}, \mathrm{Na}^{+}, \mathrm{Ca}^{2+}, \mathrm{Mg}^{2+}, \mathrm{Cl}^{-}, \mathrm{HCO}_{3}{ }^{-}, \mathrm{HPO}_{4}{ }^{2-} \mathrm{CO}_{3}{ }^{2-}$ & 1000 \\
$\mathrm{Cu}^{2+}, \mathrm{Fe}^{3+}, \mathrm{Co}^{2+}, \mathrm{Ni}^{2+}$ & 500 \\
$\mathrm{Starch}^{2-}$ glucose, citric acid, uric acid, lactic acid, & 300 \\
Pyruvic acid, cholesterol, tartrate, oxalate & 10 \\
protein (BSA), ascorbic acid & \\
\hline
\end{tabular}

isoniazid solutions) stream, the changes of the flow rate over a wide range did not result in any obvious effects on the enhancing ECL effect of isoniazid in the proposed ECL system. The reason was that these two streams provided a means to online mix luminol with isoniazid. At the same time, it was also found that the flow rate of carrier stream was other key factor for affecting the enhanced ECL signal of isoniazid in the proposed ECL flow system. The main reason was that the flow rate of carrier stream not only controlled the retaining time of the ECL emitter, which was generated electrochemically in the near surface of the electrode, in the ECL emission detector, but also provided a helping force to make an effective chemical reaction of electrochemical oxidation products of isoniazid with dissolving oxygen to produce the more active oxygen species on the near surface of electrode.

This effect was studied in the range of 0.5 to $3.0 \mathrm{~mL} / \mathrm{min}$. The results showed that a $2.5 \mathrm{~mL} / \mathrm{min}$ carrier stream flow rate was the best one and it can provide the strongest enhancing ECL signal of isoniazid. So it was selected for the subsequent experiments.

\section{Effect of the luminol concentration}

The initial testings showed that the ratio of the enhancing ECL signal of isoniazid to the blank luminol ECL signal was more dependent upon the luminol concentration; thus this effect was further studied in detail. The investigated results showed that no one of the concentrations investigated was better than that of $8.0 \mu \mathrm{mol} / \mathrm{L}$ luminol for obtaining the biggest ratio of signal to noise. Thus, $8.0 \mu \mathrm{mol} / \mathrm{L}$ luminol was selected as an optimum concentration for detecting isoniazid.

\section{Analytical performance for isoniazid measurements}

Under the selected conditions given above, the calibration curve of isoniazid standard solutions was obtained with an interval injection method. The response to isoniazid concentration was linear in the range of $4.0 \times 10^{-8}$ to $8.0 \times 10^{-6}$ $\mathrm{mol} / \mathrm{L}$ and with $2.8 \times 10^{-8} \mathrm{~mol} / \mathrm{L}$ detection limit (signal to noise $=3)$. The regression equation was $I(\mathrm{mV})=1.16+13.6$ [isoniazid] $\left(10^{-7} \mathrm{~mol} / \mathrm{L}\right)$. The correlation coefficient was 0.9998 . The relative standard deviation was less than $5 \%$ for the determination of $2.0 \times 10^{-7} \mathrm{~mol} / \mathrm{L}$ isoniazid $(n=7)$.

\section{Interference study}

The effect of common components in serum on the determination of isoniazid was studied. One species was not considered to interfere if it caused a relative error of less than $5 \%$ for the determination of $4.0 \times 10^{-7} \mathrm{~mol} / \mathrm{L}$ isoniazid. The tolerable ratios of common components in serum to $4.0 \times 10^{-7}$ $\mathrm{mol} / \mathrm{L}$ isoniazid are listed in Table 2. As can be seen from Table 2, the proposed ECL method presents the better selectivity.
Table 3 Determination of isoniazid in pharmaceutical preparation

\begin{tabular}{ll}
\hline Calibration method $^{\mathrm{a}}$ & $19.8 \pm 0.5 \mathrm{mg} / \mathrm{mL}$ \\
Standard addition method $^{\mathrm{a}}$ & $19.6 \pm 0.5 \mathrm{mg} / \mathrm{mL}$ \\
Reference value & $20 \mathrm{mg} / \mathrm{mL}$ \\
\hline
\end{tabular}

a. Average of three determinations.

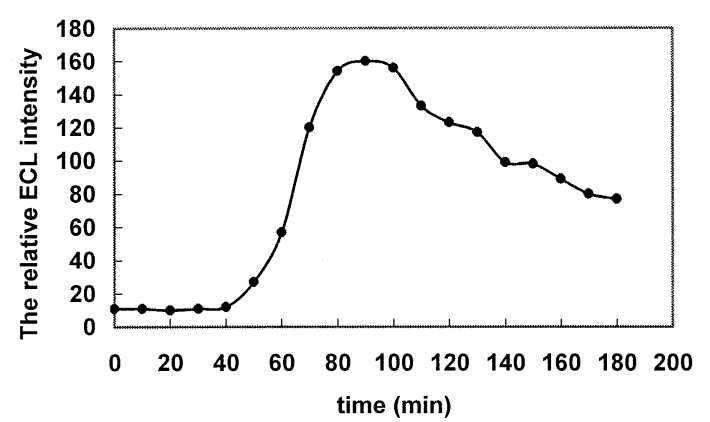

Fig. 5 The profile of the related ECL signal to the time. The flow rate of syringe pump, $5 \mu \mathrm{L} / \mathrm{min}$; the concentration of luminol, $8.0 \times$ $10^{-6} \mathrm{~mol} / \mathrm{L}$; carrier stream, $0.05 \mathrm{~mol} / \mathrm{L}$ borax buffer solution; applied potential, $0.80 \mathrm{~V}$ ( $v s . \mathrm{Ag} / \mathrm{AgCl}$ reference electrode).

\section{Analytical application}

The concentration of isoniazid in an injection as a pharmaceutical preparation was determined by the proposed method. A sample was diluted suitably with water to bring the isoniazid concentration into the response concentration range before measurement. The results are shown in Table 3. The values obtained by the calibration method as well as those obtained by the standard addition method were in excellent agreement with the reference value.

For evaluating the potential of the proposed ECL method as the better candidate to investigate the in vivo pharmacokinetic application of isoniazid, a pin-style flexible microdialysis probe (molecular weight cut-off is 5000), filled with physiological saline solution (termed the perfusate), was implanted into the edge vein of a white and awake rabbit's ear (weight: $2.5 \mathrm{~kg}$ ) by the similar way to those described previously. ${ }^{33}$

Due to the limitation of the microdialysis sampling technique on the perfusion flow rate, ${ }^{9,34}$ the dialysis species and the luminol solution was on-line mixed by a syringe pump (flow rate: $5 \mu \mathrm{L} / \mathrm{min}$ ) and a slow rate pump (flow rate: $5 \mu \mathrm{L} / \mathrm{min}$ ), respectively. Then, the on-line mixing solution in the sampling loop was injected into the flow cell every $10 \mathrm{~min}$ and the blank ECL signals were recorded. All of these operations were achieved by a Petinum II computer. After this ECL system run $1.5 \mathrm{~h}, 2.5 \times 4 \mathrm{mg}$ isoniazid was injected into muscle of this white rabbit by a $5 \mathrm{~mL}$ injector. The related ECL signals were recorded and defined as the enhancing ECL signal. The profile of the isoniazid concentration, detemined by the relative ECL signals, to the time after the drug was injected into the muscle of this white rabbit is shown in Fig. 5, and the relative ECL signals were obtained by deducting the blank ECL signal from the enhancing ECL signals. This profile revealed that the proposed method gave the better detecting performance to investigate in vivo isoniazid concentration change in the white rabbit blood. This method can be further used to investigate the pharmacokinetic process of isoniazid. 


\section{Acknowledgements}

This study was supported by the National Natural Science Foundation of China (No. 39730160).

\section{References}

1. N. M. A. Mahfouz and K. M. Emara, Talanta, 1993, 40, 1023.

2. C. S. P. Sastry, S. G. Rao, P. Y. Naidu, and K. R. Srinivas, Anal. Lett., 1998, 31, 263.

3. J. A. Garcia-bautista, J. V. Garcia Mateo, and J. M. Calatayud, Anal. Lett., 1998, 31, 1209.

4. J. A. Tong, X. J. Dang, and L. H. Li, Electroanalysis, 1997, 9, 165.

5. S. T. Sulainman and Y. O. Hameed, Anal. Chim. Acta, 1988, 206, 379.

6. F. Zhao, Y. Y. Wu, Z. Gen, and H. X. Wang, Fenxihuaxue (Chinese), 1997, 25, 927.

7. S. A. Halvatzis, M. M. Timotheou-Potamia, and A. C. Calokerinos, Analyst, 1990, 115, 1229.

8. J. Huang, C. Zhang, and Z. Zhang, Fresenius J. Anal. Chem., 1999, 363, 126.

9. M. I. Davies, Anal. Chim. Acta, 1999, 379, 227.

10. X. Paez, P. Rade, S. Tucci, N. Rodriguex, and L. Hernandez, J. Chromatogr. A, 1996, 735(1 - 2), 263.

11. M. D. Telting, O. D. Scott, and E. C. Lunte, Anal. Chem., 1992, 64, 806.

12. X. Paez and L. Hernandez, Life Sci., 1997, 61, 847.

13. A. W. Knight and G. M. Greenway, Analyst, 1994, 119, 879
14. I. Rubinstein, C. R. Martin, and A. J. Bard, Anal. Chem., 1983, 55, 1580 .

15. A. W. Knight, Tr. Anal. Chem., 1999, 18(1), 47.

16. M. Sato and T. Yamada, Anal. Sci., 1986, 2, 529.

17. A. W. Knight and G. M. Greenway, Analyst, 1994, 120, 2543.

18. R. Wilson and D. J. Schiffrin, Anal. Chem., 1996, 68, 1254.

19. Y. Wang and E. S. Yeung, Anal. Chim. Acta, 1992, 266, 295.

20. T. A. Nieman, Microchim. Acta, 1988, 3, 239.

21. J. P. Preston and T. A. Nieman, Anal. Chim., 1996, 68, 966.

22. G. B. Xu and S. J. Dong, Electroanalysis, 1999, 11, 1180.

23. S. K. Poznyak and A. I. Kulak, Talanta, 1996, 43, 1607.

24. C. A. Marquette and L. J. Blum, Anal. Chim. Acta, 1999, 381,1 .

25. V. C. Tsafack, C. A. Marquette, B. Leca, and L. J. Blum, Analyst, 2000, 125, 151.

26. K. E. Haapakka, Anal. Chim. Acta, 1982, 139, 229.

27. K. E. Haapakka and J. J. Kankare, Anal. Chim. Acta, 1980 $118,333$.

28. J. An and Q. Yao, Fenxi Huaxue (Chinese), 1990, 18, 867.

29. J. E. Vitt, D. C. Johnsen, and R. C. Engstrom, J. Electrochem. Soc., 1991, 138, 1637.

30. S. Sakura, Anal. Chim. Acta, 1992, 262, 49.

31. G. N. Chen, R. E. Lin, H. S. Zhuang, Z. F. Zhao, X. Q. Xue, and F. Zhang, Anal. Chim. Acta, 1998, 375, 269.

32. E. H. White and D. F. Roswell, "Chemi- and Bioluminescence”, ed. J. G. Burr, 1985, Marcel Dekker, New York, H.215.

33. Y. Huang, Z. Zhang, J. Lv, and H. Cheng, Anal. Chim. Acta, 2000, 419, 175.

34. I. Jacobson, M. Sandberg, and A. Hamberger, J. Neurosci. Methods, 1985, 5, 263. 